\title{
Strates
}

STRATES Matériaux pour la recherche en sciences sociales

$11 \mid 2004$

Jeune recherche, la vitalité d'un laboratoire

\section{Au-delà de la représentation, le paysage}

Sophie Bonin

\section{(2) OpenEdition}

Journals

Édition électronique

URL : http://journals.openedition.org/strates/390

DOI : $10.4000 /$ strates.390

ISSN : 1777-5442

Éditeur

Laboratoire Ladyss

Édition imprimée

Date de publication : 1 janvier 2004

ISSN : 0768-8067

\section{Référence électronique}

Sophie Bonin, «Au-delà de la représentation, le paysage », Strates [En ligne], 11 | 2004, mis en ligne le 14 janvier 2005, consulté le 10 décembre 2020. URL : http://journals.openedition.org/strates/390 ;

DOI : https://doi.org/10.4000/strates.390

Ce document a été généré automatiquement le 10 décembre 2020.

Tous droits réservés 


\title{
Au-delà de la représentation, le paysage
}

\author{
Sophie Bonin
}

1 En réfléchissant sur l'évaluation des paysages de la Loire faite à l'occasion de ma thèse de doctorat, j'ai été confronté à plusieurs questions :

2 - quelle est la place de la Loire dans l'espace vécu des riverains? Comment la considèrent-ils? Quel rôle joue-t-elle dans les comportements? Est-elle valorisée, banalisée ou occultée? En somme, quelles sont leurs représentations mentales du fleuve?

3 - quel est l'effet des discours, représentations publiques, sur les conceptions de la Loire: dans quelle mesure transforment-ils les représentations que les habitants construisent de ce fleuve proche?

4 Parties d'une interrogation sur le thème du paysage, ces questions ne recourent pas une fois au terme de paysage mais à celui de représentation. Ceci m'a conduite à mener des investigations, par rapport à la géographie, sur ces deux mots. Le premier constat, pour ne pas dire la première impression, a été que beaucoup de géographes, intéressés par la géographie humaine et les sciences sociales, ont eu, à un moment ou à un autre, à utiliser ces termes. Mais cette recherche avait un objectif clair, qui n'était pas épistémologique, mais simplement une quête d'un corpus théorique et méthodologique qui puisse aider à répondre aux questions concrètes évoquées.

5 C'est alors qu'est apparu l'intérêt de plusieurs courants développés récemment de la géographie comme science sociale. Un nouveau chantier de défrichement s'est ouvert, depuis les années soixante-dix, selon deux axes, qui se sont prolongés en s'ignorant dans une large mesure, mais qui ont en commun de développer une géographie très humaine, humaniste, reposant l'homme au centre de l'analyse des espaces. L'un de ces axes a remis au goût du jour des concepts nés en sociologie, en particulier dans la psychologie sociale, pour tenter une géographie des représentations. L'autre a plutôt émergé de réflexions philosophiques sur l'esthétique et la modernité, pour construire ce que l'on appellera ici une théorie du paysage, en référence à un ouvrage d'une des personnalités, sinon de la personnalité dominante de ce mouvement, Alain Roger ${ }^{1}$. Cet 
ouvrage, qui est une anthologie de textes théoriques sur le paysage, d'époques diverses, d'auteurs issus de disciplines très différentes parfois, ne propose pas à proprement parler la «théorie du paysage » annoncée par le titre. Mais tous ces textes ont, malgré leur diversité, en commun un point de vue théorique sur le paysage, que nous développerons plus loin, et qui insiste sur l'idée du paysage comme construction sociale, ou culturelle, cognitive au sens large, plus que sur celle du paysage comme étendue terrestre (qui n'est pas pour autant négligée par certains des textes de cette anthologie).

6 Ces deux axes prennent de l'importance dans la littérature scientifique durant la même période, le milieu des années soixante-dix, mais de façon autonome si on s'en tient aux auteurs concernés et aux deux réseaux de références construits par eux. D'ailleurs, le deuxième mouvement s'est dans une large mesure développé en dehors de la géographie, bien que son objet soit le rapport au territoire. Sur les idées, un certain nombre de définitions de concepts (représentation d'un côté, paysage de l'autre), une même conception de ce qu'est la connaissance, et une même préoccupation pour la subjectivité du rapport au territoire poussent à chercher à les réunir.

7 Cet article se propose de rassembler les éléments des débats qui éclairent mutuellement ces deux mouvements de la recherche géographique française, dans une perspective bibliographique. Certaines de ces recherches ayant presque trente ans, le recul est sans doute suffisant pour examiner pourquoi ces deux mouvements, en quelque sorte, sont restés séparés, et quel intérêt il y a à les rapprocher. De façon pratique, l'espoir est grand de voir un jour cesser un usage abusif des termes de représentation et de paysage chez certains géographes, qui font fi de la considérable littérature produite sur ces sujets. Il ne s'agit pas d'arrêter des définitions, mais de lancer un appel à une plus grande considération des débats actuels sur ces notions, et leur confrontation.

8 L'axe de recherche sur les représentations en géographie sera d'abord présenté, puis celui sur une "théorie du paysage ». Nous tenterons dans une dernière partie de faire la synthèse des deux à travers les apports de l'étude des représentations pour celle des paysages.

Géographie et représentations

9 Le terme de représentations spatiales, qui peut paraître indispensable dans l'appréhension scientifique du paysage, a été particulièrement traité par un groupe de géographes, et précisé, en 1984, dans l'introduction de la thèse de Jean-Paul Guérin. Celui-ci définit le mot discours en se référant aux sciences cognitives comme «création sociale [ou individuelle ajoutera Antoine Bailly plus tard] de schémas pertinents du réel »: cette définition va servir de référent pour le terme de représentation aux auteurs défendant une "géographie de la perception" (Bailly, 1974), qui sans changer d'objet véritablement, deviendra "géographie des représentations " (Bailly, 1985). Les principaux auteurs moteurs de ce mouvement sont, en plus d'Antoine Bailly ou JeanPaul Guérin, Hervé Gumuchian, Bernard Debarbieux, Robert Ferras, Claude Raffestin, Yves André. La bibliographie est riche, en particulier grâce à une série d'ouvrages collectifs : publication des actes du colloque de Lescheraines (Guérin et Gumuchian, 1985) et de l'université d'été de 1989 à Sérignan-Plage (André, Bailly, Clary, Ferras, et Guérin, 1990). Cette série s'achève avec un ouvrage d'Yves André, qui tente une synthèse des travaux précédents, et développe le concept de représentations spatiales dans un but didactique (André, 1998). L'expression de géographie des représentations a 
été discutée par les auteurs eux-mêmes, et le glissement de terme effectué par Antoine Bailly de perception vers représentation était déjà issu d'âpres critiques sur ce thème ${ }^{2}$. Elle porte en effet à confusion puisqu'il ne s'agit pas tant de faire une géographie des représentations que d'étudier les représentations géographiques, voire, si le terme ne paraissait pas trop ambitieux, il s'agit de l'étude des géographies. Je propose ici la terminologie de géographie représentationniste qui a le mérite d'éviter la confusion évoquée: cette géographie n'a pas de particularité dans ses objets d'étude mais seulement dans la façon de les aborder. Elle présente deux inconvénients qu'il faut préciser: d'une part c'est un néologisme, d'autre part cet adjectif est utilisé par les linguistes dans un sens qui leur est propre. Toujours est-il que ces recherches travaillent à l'étude des représentations de l'espace, et le terme de paysage est en général soigneusement évité. Cette absence n'est pas inattendue dans certains travaux qui restent très proches de la psychologie et des sciences cognitives (Bailly, Raffestin, et Reymond, 1980), mais même ces travaux approchent parfois des thèmes paysagers. Par exemple Antoine Bailly (1985), en travaillant sur les problèmes d'orientation de l'individu et d'appréciation des distances, parle de modèles historiques et culturels par rapport à des propriétés symboliques. Les choses sont encore plus claires lorsqu'il conclut sur les trois aspects de l'espace mental : structurel, fonctionnel et symbolique, détaillant les deux premiers termes, mais laissant délibérément de côté le troisième aspect « moins souvent abordé, il résulte du caractère relationnel de la représentation et de la variété des expériences spatiales individuelles ». On retrouve là le même argument qu'utilisent beaucoup de géographes pour justifier leur méfiance face au thème du paysage comme représentation: la variété (pour ne pas dire l'infinité) des expériences possibles. Une autre hypothèse quant à l'évitement du mot paysage est la volonté de ne pas reprendre un terme très chargé de significations pour la géographie : il est difficile d'utiliser un mot très marqué par l'usage traditionnel, celui de la géographie régionale, de Marc Bloch, de Roger Dion ou, plus près de nous, de JeanRobert Pitte ${ }^{3}$.

Ces travaux se rapprochent également du thème du paysage lorsqu'ils utilisent le terme d'image. Le terme image (mentale ${ }^{4}$ ou non) semble en effet souvent substituable à celui de représentation. Par exemple Clara Copeta, dans le colloque de 1985, donne cette définition des représentations, «empruntée » à la sémiologie et à la philosophie : «la Représentation ou Image est le résultat d'un processus qui a comme base le perçu » (Copeta, 1985, p. 15).

11 Enfin les recherches en matière de représentations adoptent toutes des présupposés philosophiques, que l'on retrouvera (mais pas à l'identique) avec la «théorie du paysage ", quant à la nature de la connaissance : s'appuyant en particulier sur Edgar Morin $^{5}$, Yves André affirme nécessaire « d'abandonner l'idée communément ressentie que la connaissance de la réalité est antérieure à la vérité et admettre que toute réalité est cernée et reconnue par la raison, qui en élabore des représentations. » (André, 1998, p. 32). Reconnaitre cette subjectivité de la connaissance l'amène à affirmer : "Toute la problématique des représentations en géographie repose donc sur l'idée que l'espace des hommes n'est pas objectif, ni rationnel (au sens d'une rationalité positiviste) : il est une reconstruction mentale et, par conséquent, une représentation spatiale ». La "théorie du paysage " se situe dans le même champ en reconnaissant le paysage comme construction mentale, mais, en particulier sous la plume d'Augustin Berque, 
elle réfute cette seule affirmation : l'espace des hommes est aussi un donné, implique aussi un « en soi » des choses.

Mais mettre au cœur de ces recherches géographiques le concept de représentation signifie se rattacher à une généalogie bien éloignée de celle du paysage : c'est un concept classique ${ }^{6}$. Il a d'abord été fort travaillé en sociologie par la psychologie sociale : concept de représentation collective «élaboré par Durkheim à la fin du XIX ${ }^{\mathrm{e}}$ siècle, revivifié par S. Moscovici au cours des années soixante » (André, 1998, p. 14). Il l'a aussi été par la psychologie cognitive et la sémiotique (Piaget, Brunner, Bachelard). Il faut signaler enfin que c'est un concept qui, en géographie, a été fortement influencé ou du moins appelé par la notion d'espace vécu d'Armand Frémont (colloque sur le sujet en 1976 à Rouen et, bien entendu, La Région, espace vécu ${ }^{7}$ ), qui a conduit à deux ouvrages sur Pratique et perception de l'espace ${ }^{8}$ en 1983 et 1984. Les objectifs des géographes qui ont fait appel à ce concept de représentation dans ces dernières années sont aussi assez éloignés des préoccupations paysagères: il s'agit parfois d'une réflexion sur les cartes, et leur usage en géographie ${ }^{9}$, souvent d'une réflexion didactique sur l'enseignement de la géographie ${ }^{10}$, enfin il s'agit souvent également d'une recherche épistémologique pour sortir de ce qui serait une crise de la géographie $^{11}$, dans l'enseignement secondaire.

Ces éléments sont importants pour justifier que ce courant de la recherche se soit développé de façon assez indépendante des recherches sur le paysage. Mais avant d'aborder les méthodes et résultats apportés par cette géographie représentationniste, susceptibles d'enrichir les approches du paysage, il convient maintenant de préciser l'autre courant déjà évoqué : celui se rattachant à la « théorie du paysage ».

Les définitions de la " théorie du paysage »

Si le recul est aujourd'hui peut-être insuffisant, il semble que le domaine du paysage, compris comme le rapport sensible des sociétés à leur territoire, ait été très fertile pour la recherche fondamentale dans les sciences humaines de ces dernières années. Même s'il est fait ici allusion à des travaux souvent réalisés par des non géographes, ce critère ne semble pas suffisant pour les rendre inintéressants à cette discipline : ils ont tous, au cœur de leur démarche le "dessin de la Terre». On est ici autant en anthropologie qu'en géographie, et c'est un philosophe braconnant, selon ses propres termes, sur les terres du paysage, des géographes et des architectes, qui a le plus enrichi ce domaine. Il lui a surtout apporté des bases conceptuelle et méthodologique solides : la théorie de l'artialisation d'Alain Roger (1978) est un point commun implicite ou explicite à ces travaux, ou en tous cas est la thèse approfondissant les tenants théoriques de cette trame de pensée sur le paysage. Cette véritable théorie du paysage propose le schéma du passage du pays - une étendue terrestre qui n'est ni désignée comme paysage, ni support de valeurs esthétiques - en paysage : c'est par le truchement de l'art, la peinture et la littérature essentiellement, que ce passage se fait. Alain Roger (1997), de façon concentrée mais aussi recentrée, en refait la démonstration dans son Court Traité $d u$ Paysage, avec un certain nombre d'exemples d'accès de nouveaux territoires au rang de paysage. Divers ouvrages publiés depuis la fin des années quatre-vingt viennent conforter cette théorie, par exemple avec l'histoire du « sentiment paysagiste » depuis le XVIII ${ }^{\mathrm{e}}$ siècle (Luginbühl, 1989b). D'autres travaux, tout en reprenant cette « fonction socio-transcendentale ${ }^{12}$ » de l'art dans la sensibilité aux paysages, y ajoutent le rôle des scientifiques et des formes de culture contemporaines, photographie, cinéma, médias multiples, qui offrent, parfois, un renouvellement du regard sur certains espaces : la 
campagne (Camporesi, 1995; Marié et Viard, 1988), la montagne (Briffaud, 1994 ; Debarbieux, 1993 ; Joutard, 1986), la plage (Corbin, 1988), récemment, le désert (Roux, 1996), ou même des villes (Vallat, Marin et Biondi, 1998). Ces travaux énoncent tous l'idée de naissance, d'invention, à propos de cette reconnaissance d'un territoire comme paysage: il ne peut dès lors s'agir que d'une facette de l'ensemble des représentations du territoire. Il semble inutile de remettre en cause la théorie de l'artialisation (peu d'auteurs s'y sont osé sérieusement ${ }^{13}$ ), mais elle ne correspond qu'à une certaine définition du paysage: précisément, le paysage artialisé... D’autres rapports, affectifs et même esthétiques, liés à une mémoire personnelle singulière ; d'autres représentations, par exemple liées à des usages économiques particuliers ou à un contact quotidien avec un territoire, ne sont pas abordables dans ce cadre. Ceci n'enlève rien au formidable champ de recherches qu'a pu ouvrir la théorie de l'artialisation: en particulier parce que le paysage artialisé est une facette qui a l'avantage d'être conçue et perçue comme collective, partageable au sein de la société ; c'est aussi une facette qui se traduit très matériellement à travers le tourisme, déterminant le code d'un ensemble de pratiques constituant un véritable rituel social ${ }^{14}$. Il semble que les géographes n'aient pas pris la mesure de l'intérêt de ces divers travaux pour la compréhension d'un phénomène aussi géographique que le tourisme.

Par rapport aux travaux évoqués sur les représentations, on se rend bien compte que le paysage, entendu ainsi, est un type particulier de représentations, une "création sociale » particulière de schémas de pensée du réel géographique. La théorie du paysage permet l'étude objective d'une catégorie de représentations, celle des paysages artialisés, ou des paysages culturels. Le terme de paysage culturel conviendrait très bien pour ces représentations issues de la culture dominante; mais il y a un risque d'amalgame avec la notion de Kulturlandschaft qui désigne un territoire où les activités humaines, en particulier celles du passé, sont particulièrement visibles. Le désert par exemple n'est pas un Kulturlandschaft, mais c'est un paysage culturel majeur en Europe...

16 Notre question devient donc plus précise: que peut bien apporter la géographie représentationniste à la théorie du paysage?

Résultats et intérêt de la géographie représentationniste pour la théorie du paysage

L'intérêt de la géographie représentationniste pour l'analyse du paysage se manifeste de trois façons : par les catégories spatiales explorées qui sont différentes (l'urbain en particulier) ; par les méthodes qui ont été expérimentées ; par les débats auxquels elle a donné lieu et qui interrogent aussi la théorie du paysage.

Catégories spatiales explorées

18 La géographie représentationniste a obtenu des résultats remarquables dans certaines catégories spatiales restées en marge de la plupart des études sur le paysage: en particulier l'urbain est un domaine privilégié de ces recherches. Les travaux sur la « configuration cognitive de l'espace » ont même d'abord et surtout porté sur l'espace de la ville, son appréhension par l'habitant en terme d'orientation, d'appréciation des distances, de " cartes mentales », en bref sur les aspects structurels et fonctionnels de l'espace mental; beaucoup moins sur les aspects symboliques. Ces recherches s'inspirent donc des développements des sciences cognitives : ce sont les processus en œuvre dans les représentations que l'on vise, plus que le contenu des représentations. Ce sont les travaux de Kevin Lynch, de A. Metton et M. J. Bertrand (1972), de Sylvie Rimbert (1973) pour n'évoquer que les précurseurs les plus cités ${ }^{15}$. On retiendra de ces 
recherches sur les processus en œuvre la mise en évidence (Bailly, 1985, p. 199) d'un référentiel égocentré (lié en particulier aux pratiques individuelles de l'espace) et un référentiel exocentré (lié aux autres représentations existantes sur ce même espace ou type d'espace $\left.{ }^{16}\right)$. Ces avancées permettent d'espérer dans l'avenir une étude qui prendrait à la fois en compte, et même confronterait ces aspects «structurels et fonctionnels ", particulièrement bien saisis par les cartes mentales, et les aspects symboliques, par exemple tels qu'on les appréhenderait avec la théorie de l'artialisation.

La géographie représentationniste a enfin permis d'insister sur l'importance de la territorialité comme phénomène psychologique.

Méthodes expérimentées

D'une façon globale, la géographie représentationniste s'est en général attachée à développer les méthodes permettant l'approche des usagers, par l'espace vécu. C'est l'idée d'une «microgéographie des représentations », géographie par le bas, qui adopte le « référentiel-habitant ${ }^{17}$ ». Les cartes mentales dont on a parlé sont un bon exemple, d'autant qu'elles n'ont pas été développées seulement en ville : Bernard Debarbieux, Yves André, Michelle Masson ont proposé une exploitation originale de la réalisation de cartes mentales par les habitants d'une vallée, d'une région, ainsi que l'analyse de dessins d'enfants. Le problème de ces méthodes est qu'elles nécessitent des précautions très importantes: d'abord parce que l'analyse d'une image n'est jamais simple, on la maitrise bien moins que l'analyse d'un texte ; ensuite parce que la nécessité de prendre en compte l'influence du sexe, du groupe social, de la profession, de l'origine de la personne essouffle la recherche quant au territoire lui-même. Une limite nette doit aussi être donnée à ces cartes : elles sont en général surtout utiles pour le problème de l'appréciation des distances, de l'orientation, qui sont fonction de la proximité et de la fréquentation. Elles ne permettent pas d'aborder d'autres éléments de la mise en forme du territoire dans les représentations. Les enquêtes et l'analyse de discours constituent donc un outil souvent jugé plus efficace, et surtout ces techniques bénéficient de l'apport considérable des recherches en sociologie. D'ailleurs certains sociologues se sont intéressés à la problématique des représentations sociales appliquées à l'environnement : c'est le cas par exemple d'un groupe de sociologues réunis autour de Nicole Eizner (1994), qui applique la méthode de Pierre Vergès à propos des représentations sociales de l'économie. Ainsi des protocoles d'enquêtes, d'analyse de contenu avec opérations statistiques, sont affinés, permettant en particulier d'utiliser des opérations cognitives connues (sélection, association, regroupement). L'élaboration des questionnaires est aussi mieux maîtrisée grâce à ces recherches (voir par exemple, Diénot, 1985). Des outils comme l'analyse de contenu sont introduits en géographie (par exemple, Collado, 1985). Enfin des sources nouvelles (pour les géographes) de discours sur l'espace sont pris en compte: les guides et la publicité touristiques en sont un fertile exemple (par exemple, Dewailly, 1984 ; Mormont, 1985 ; Tourneux, 1987).

D'autres méthodes enfin ont été testées par des études sur les représentations, et ont inspiré récemment les méthodes d'étude du paysage: enquêtes par classement de dessins, de photographies, tests associatifs, sont évoqués par Antoine Bailly en 1985. On les retrouve dans les études sur le paysage à partir de série de photos ${ }^{18}$, de listes de mots à associer, jusqu'à la réalisation spontanée de photos par les enquêtés ${ }^{19}$.

Débats animés 
De vifs débats ont été animés et publiés à propos de la géographie des représentations, dont certains éléments sont très utiles lorsque l'on envisage une recherche à partir de la théorie du paysage. La complexité des liens entre l'objet et sa représentation est le principal obstacle à une étude scientifique des représentations. Une grande part des critiques porte donc sur le problème du medium qui est analysé, à défaut de pouvoir saisir autre chose: on ne peut saisir que l'expression, la forme d'une représentation (que ce soit une image, un dessin, une photo, ou bien un discours spontané, un texte littéraire, un rapport d'étude). Or il y a deux écueils. D'une part cette expression a un poids, une autonomie qui rejaillit sur la représentation elle-même (Guérin, 1990) : la conjoncture de tout discours, celle qui le détermine et sur laquelle il intervient, a une influence considérable sur les réponses qui sont données lors d'enquêtes. D'autre part une représentation n'est qu'un produit possible du processus complexe qui conduit de l'objet à sa perception, puis à son interprétation, et enfin seulement, éventuellement, à son expression. Ainsi Jean-Paul Guérin (1984) évoque le jeu entre discours et perception (le premier n'englobe pas tout le second; le premier peut générer le second sur un mode virtuel). Et un degré supplémentaire de complexité est atteint lorsque, ce qui est souvent l'objectif de la recherche, on considère que l'enjeu des discours est dans les comportements, les pratiques, c'est-à-dire l'utilisation de l'espace. Ce dernier rouage est celui qui a peut être été le plus étudié : la mesure des écarts entre discours et pratiques est un classique en matière d'étude des loisirs (par exemple, Debarbieux, 1985 ; Diénot, 1985 ; Kalaora, 1993 ) ; le lien entre représentations et aménagement est plus une «spécialité » des géographes (Berque, 1995 ; Guérin, 1984 ; Piolle, 1985²0). On arrive ainsi à l'étude, particulièrement intéressante pour le paysage, de la diffusion des représentations en rapport avec l'affirmation des groupes sociaux (c'est-à-dire leurs pratiques). C'est le rôle de la distinction selon Bourdieu, qui peut expliquer comment des représentations développées par quelques individus deviennent dominantes : «le sujet inscrit dans le réel la syntaxe de son propre discours, celui de sa classe socioéconomique " (Bailly, 1974, p. 212); et certains vont plus loin affirmant "l'impossible définition de systèmes de représentations clos et immanents aux groupes qui les portent. Les représentations parce qu'elles sont mises en pratique se redéfinissent constamment, définissent les groupes et deviennent motrices de l'inscription des groupes dans la société " (Boumaza, 1985, p. 161). Ce genre de proposition peut se concevoir comme un argument explicatif de la théorie du paysage, de cette diffusion de modèles paysagers d'abord générés au sein d'un groupe très étroit. Mais là encore, il ne rend pas compte de l'ensemble des représentations du paysage, mais seulement des plus dominantes dans la société.

Il faut revenir au problème dénoncé : «Quel dédale sépare du réel appréhendé l'image du vécu!» (Hussy et Lopreno, 1985, p. 314), conduisant à prendre les cartes mentales comme des utopies. Il est en quelque sorte résolu par la nécessaire distinction à faire entre réalité et image mentale : ce sont « deux conceptions distinctes régies, l'une par des critères de vérité (et donc essentielle), l'autre de pertinence (et donc existentielle) » (p.323). C'est dans ce deuxième champ que l'on doit situer toute étude sur les représentations. C'est pour cela également que l'utilisation des représentations dans l'enseignement, lorsqu'elle consiste à dénoncer des représentations «fausses » par rapport à un savoir scientifique "vrai ", aboutit à une impasse. De même l'intérêt des analyses qui se focalisent sur la distorsion des représentations avec les données physiques, objectives, est vite limité. Enfin, une autre mise en garde est que l'on ne peut pas analyser sur le même plan différentes caractéristiques des représentations : celles- 
ci sont «fonction de qualités propres à l'homme, telles que le souvenir et l'imagination, que l'on ne peut évaluer, ni quantifier, et d'autres qualités propres à l'objet de la représentation, telles que la fonction sémiotique et celle symbolique » (Copeta, 1985, p. 15). Il en ressort la possibilité de l'étude, et même la priorité à donner à celle du symbole qui «permet de nommer l'expérience ou encore de l'organiser, donc, de la constituer en tant que telle; il permet aussi de la rendre concevable et communicable ».

Conclusion

Cet article, s'il ne clôt pas les débats respectifs qui animent ces deux courants, la géographie représentationniste et la théorie du paysage, a montré les avancées méthodologiques qu'ils offrent, et éclairé sous un certain angle l'utilisation des termes de paysage et de représentation.

Considérant en particulier l'usage, classique et durable, du terme de paysage par les géographes, il semblerait utile de préconiser le terme représentation du paysage pour désigner ce que les anthropologues et les théoriciens nomment paysage, en étant généralement très mal compris des géographes...

La possibilité de rapprocher ces deux thèmes de recherche dévoile l'intérêt que pourrait avoir, pour des géographes, l'orientation de travaux de recherche à partir de la théorie du paysage, bien que celle-ci ne soit pas, à l'origine, géographique. Elle se présente comme une spécialisation possible de la géographie représentationniste. Dans tous les cas, ces recherches témoignent d'un intérêt nouveau pour la prise en compte des connaissances rationnelles ou non (sciences, littérature, art, médias, discours) des territoires. La position proposée ici ne manquera pas de choquer ceux qui considèrent le thème des représentations dépassé, en particulier parce qu'une représentation apparaît souvent comme un reflet caricatural de la réalité, et non comme une médiation complexe. On peut prétendre dépasser ce reflet caricatural, et se rapprocher de la compréhension de la médiation complexe, en y ajoutant simplement une place primordiale à la notion de pertinence, comme le propose Jean-Paul Guérin et les géographes qui ont adopté sa définition. En effet, il est nécessaire de mettre au cœur de l'analyse cette notion: pertinence des représentations entre elles, et avec l'étendue terrestre qui les provoque et qui les soutient.

Revenant à ma thèse de doctorat de géographie, sur l'évaluation des paysages de la Loire, c'est au croisement de ces deux thèmes que j'ai trouvé les plus solides bases théoriques et méthodologiques. Ce sont elles qui m'ont permis d'accepter la multitude des sources de représentations sur le fleuve, textes, discours ou images. En particulier, elles m'ont amenée à remettre en cause le point de vue classique des recherches issues de la théorie du paysage, qui consiste à analyser les discours et images rendus dominants (en ne prenant donc les discours actuels seulement en ce qu'ils confirment un modèle). Le travail de recherche a au contraire cherché à partir de discours actuels, en particulier d'habitants riverains, en les considérant, autant que possible, comme originaux (ce qui nécessite, mais seulement comme préalable, d'analyser les discours dominants). Cette approche cependant conduit assez vite à ne plus tant parler de paysage que de « rapport » à un territoire, et à voir émerger des groupes de sensibilité liés surtout à des pratiques de ce territoire (y compris et surtout l'observation). Elle rend difficile la recherche de modèles de paysage, d'une quelconque «invention du paysage ", mais met en évidence un processus fondamental de métonymie : un objet (un arbre, une mare, une usine) du territoire fait paysage, c'est-à-dire que c'est à partir 
de cet élément que se tisse un rapport sensible au territoire. Dès lors étudier les paysages de la Loire, ce n'est pas étudier le paysage vu de Candes, celui du Val d'Or de Saint-Benoît ou du Bec d'Allier, mais plutôt le paysage du saule, celui du saumon, celui des crues ou de l'abaissement du plan d'eau, celui du noyé ou celui de la pollution. Autrement dit, l'analyse de discours actuels sur le paysage, sur la relation sensible de "simples gens", d'agents sociaux comme diraient certains, au territoire, ramène l'analyse à une dimension matérielle. Aussi, même le concept de représentation tel que nous l'avons présenté, se trouve-t-il dépassé, ou à dépasser en tous cas : il ne suffit pas à appréhender la relation affective que les hommes tissent avec leur espace. Ce sont les " modes de voir » qui sont débordés par les «modes d'habiter ». Ce constat fait dans la thèse à partir d'une centaine d'entretiens, longs, avec des riverains de la Loire, nous a conduite à chercher d'autres notions. Pour à la fois conserver les acquis de ces recherches sur le paysage comme construction sociale et culturelle, voire individuelle, et sur les représentations, nous nous sommes rappelés les « affinités électives » si bien évoquées, voire définies par Goethe. Pour rendre cette notion dans une problématique géographique, lui conserver ce qu'elle suggère comme tension depuis l'homme d'une part, et depuis la nature d'autre part, nous avons eu recours à l'expression de préférences paysagères. Conduire une "géographie des préférences paysagères ", le long de la Loire, est ainsi devenu notre gageure. Il est entendu que nous n'avons pu que l'esquisser. L'étendue du terrain à défricher paraissait plus grande à chaque mètre du fleuve : mais après tout, quelle plus belle découverte pour des géographes?

\section{BIBLIOGRAPHIE}

André Y. (1998). Enseigner les représentations spatiales, Anthropos-Economica.

André Y., Bailly A., Clary M. et al. (1990). Modèles graphiques et représentations spatiales, AnthroposReclus.

Bailly A. (1974). « La perception des paysages urbains - Essai méthodologique » dans L'Espace géographique, III (3), p. 211-217.

Bailly A. (1985). « Distances et espaces : vingt ans de géographie des représentations » L'Espace géographique, XIV (3), p. 197-205.

Bailly A. (1995). « Géographie régionale et représentation » in A. Bailly, M. Bernard et B. Debarbieux (dir.), Géographie régionale et représentations, Anthropos, p. 25-34.

Bailly A., Raffestin C., Reymond H. (1980). « Les concepts du paysage : problématique et représentations » L'Espace géographique, IX (4), p. 277-286.

Berque A. (1995). Les raisons du paysage - de la Chine antique aux environnements de synthèse, Hazan. Boumaza N. (1985). « Questions de représentation dans l'immigration d'origine étrangère en France » in J.-P. Guérin et H. Gumuchian (dir.), Les représentations en actes - Actes du colloque de Lescheraines. Grenoble, Institut de Géographie Alpine - Université de Grenoble, p. 145-162. 
Briffaud S. (1994). Naissance d'un paysage - La montagne pyrénéenne à la croisée des regards, XVI ${ }^{e}$-XIX siècles, Association Guillaume Mauran, Archives des Hautes-Pyrénées, CIMA-CNRS, Université Toulouse II.

Brunet R. (1990). « À quoi sert la chorématique » in Y. André, A. Bailly, M. Clary, R. Ferras et J.-P. Guérin (dir.), Modèles graphiques et représentations spatiales, Anthropos - Reclus, p. 27-39.

Camporesi P. (1995). Les belles contrées - Naissance du paysage italien, Gallimard.

Cazes G., Knafou R. (1995). « Le tourisme » in A. Bailly, R. Ferras et D. Pumain (dir.), Encyclopédie de géographie ( $2^{\mathrm{e}}$ éd.,), Economica, p. 827-844.

Collado M. (1985). «Étude du discours portant sur les définitions des enjeux liés aux petites hydrocentrales électriques » in J.-P. Guérin et H. Gumuchian (dir.), Les représentations en actes Actes du colloque de Lescheraines. Grenoble, Institut de Géographie Alpine - Université

\section{NOTES}

\section{A. Roger, 1995.}

2.Le terme de perception convient mal en effet à la géographie, étant en général délimité comme un « acte instantané de nature physiopsychologique ", c'est-à-dire qui fonctionne exclusivement en présence de l'objet par l'intermédiaire d'un champ sensoriel (C. Hussy et D. Lopreno, 1985, p. 318).

3.J.-R. Pitte (1986). Histoire du paysage français, Tallandier.

4.L'expression d'image mentale a été développée par exemple dans A. Bailly, 1985.

5.E. Morin (1986). La connaissance de la connaissance : anthropologie de la connaissance, Seuil. 6.On peut relever que certains auteurs, comme C. Hussy et D. Lopreno (1985), font remonter les premiers travaux de « psychologie de l'espace » à 1939 (G. Hardy, Géographie psychologique, Gallimard), 1944 (W. Hellpach, Géopsyché, Payot) ou 1946 (A. Arnoux, Géographie sentimentale, Lardanchet).

7.A. Frémont, La Région, espace vécu, $2^{\mathrm{e}}$ éd., Champs Flammarion, 1999. On y retrouve l'absence de développement utilisant le mot paysage, mais une large utilisation de celui de représentation.

8.Institut de Géographie de Neuchâtel (1983). Pratique et perception de l'espace Bulletin de la Société Neuchâteloise de géographie, no 27 ; et UA CNRS 911, université de Pau (1984). Actes de la table-ronde à Pau, 6 au 8 septembre 1984 - Revue Hegoa, no 1.

9.C'est en particulier la participation de Roger Brunet à ce débat : « Les modèles d'espaces que nous produisons [les chorèmes] sont des représentations d'organisations réelles, telles bien entendu que nous les comprenons. Ces organisations sont imaginées, mais non imaginaires. Il ne faut pas confondre en effet image, imagination et imaginaire » (R. Brunet, 1990, p. 32). Voir aussi sur ce sujet R. Ferras, 1993. L'idée de la carte comme représentation et des conséquences de ce rapprochement sont bien développées dans le même ouvrage (G. P. Torricelli, 1990, p. 101) : «'Le voile symbolique' c'est le dessin de la Terre, la carte géographique, mais elle ne peut être séparée d'une autre forme de représentation [...] : la peinture de paysage, qui par définition est une image vue à travers les yeux du peintre. Attention, semble nous dire Humboldt; quand nous regardons un tableau ou nous écoutons une description de paysage, nous savons tous qu'il ne s'agit que d'une représentation personnelle (d'un point de vue comme aurait dit Ritter) ; par contre, quand on regarde une carte, nous 
oublions souvent qu'il s'agit également d'une représentation, et que son code dépend du point de vue de l'auteur. "

10.Ce souci est particulièrement au cœur des ouvrages collectifs déjà évoqués, ainsi que dans Y. André, 1998 et M. Masson, 1995.

11.Voir par exemple Michelle Masson évoquant les professeurs « affrontant le désintérêt des élèves pour une matière qui répond mal à leurs questions, concurrencée par les photographies en couleur des magazines, les images télévisées et la syntaxe des médias. Il y a crise de l'enseignement de la géographie. » (M. Masson, 1995, p. 2). Yves André ou Hervé Gumuchian ont exprimé la même préoccupation.

12.A. Roger, 1978.

13.L'article de Jacques Dewitte (1998) est, selon nous, un perfectionnement dans l'explication de la théorie d'Alain Roger, et non une remise en cause. Jacques Dewitte y montre que l'idée d'une « structuration immanente du monde », d'une beauté du monde qui précède l'homme, n'est pas contradictoire avec l'idée d'artialisation, et même que "si l'idée d'artialisation a un sens, elle a forcément à voir avec l'amour ", p. 365. L'article finalement critique « l'artificialisme » dont Alain Roger accompagne sa théorie, et non celle-ci.

14. Voir la définition du touriste comme « tout le monde, ou presque, à un moment ou à un autre » dans G. Cazes, et R. Knafou, 1995, p. 829.

15.Deux articles offrent une bonne présentation bibliographique sur ce sujet : A. Bailly, 1985 ; B. Debarbieux, 1985.

16.Dans cet article, Antoine Bailly considère comme référentiel exocentré les cartes, par exemple un plan de ville. Cette définition a été élargie à partir de A. Bailly, 1995. L'auteur y reprend la notion de référentiel égocentré en l'associant à l'espace vécu, alors que le référentiel exocentré est associé aux espaces représentés, par les connaissances indirectes (médias).

17.L'expression est de Jean-Paul Ferrier (voir à ce sujet H. Gumuchian, 1988, p. 21).

18. Voir sur ce sujet : Y. Luginbühl, 1989a.

19.Méthode originale développée par Yves Michelin, en particulier appliquée sur la montagne Thiernoise, à la fin des années quatre-vingt-dix (l'auteur utilise ouvertement les recherches en matière de représentation).

20.Ce qui conduit Xavier Piolle à s'intéresser aux rapports entre représentations et pouvoir : «Considérer l'aménagement comme une mise en actes de représentations collectives nous conduit à nous interroger sur la validité, comme champ de recherche, de ce champ d'intervention dont le pouvoir politique fait son domaine réservé » (p. 94).

\section{RÉSUMÉS}

La géographie comme science sociale a récemment vu se (re)développer deux problématiques originales, qui ont en commun de montrer une géographie très humaine, humaniste, reposant l'homme au centre de l'analyse des espaces. L'une a remis au goût du jour des concepts nés en sociologie, en particulier en psychologie sociale, pour tenter une géographie des représentations. L'autre a plutôt émergé des réflexions philosophiques sur l'esthétique et la modernité, pour 
construire une véritable théorie du paysage. Cet article tente de rapprocher ces deux problématiques, qui émergent de la littérature scientifique vers la même période, le milieu des années 70, mais de façon autonome si on s'en tient aux auteurs concernés et aux deux réseaux de références construits par eux. Car sur les idées, un certain nombre de définitions de concepts (représentation d'un côté, paysage de l'autre) et une même préoccupation pour la subjectivité du rapport au territoire forcent à chercher à les réunir. L'objectif inavoué est d'alerter sur l'existence d'une abondante littérature quant à l'usage respectif des termes de représentation et de paysage, dans l'espoir de voir un jour cesser des emplois souvent abusifs...

Two original thematics have recently (re)emerged in French geography: the geography of representations and the theory of landscapes. Both put man at the core of the analysis of spaces, and throw light on a very human, even humanistic, aspect of geography. The geography of representations is based on concepts coming from sociology, and more precisely, social psychology. The theory of landscapes derives from philosophical reflections on esthetics and modernity. This paper intends to link both theories, which appeared at the same period (mid 70s), but grew separately. However, certain definitions of concepts ("representation" on the one hand, "landscape" on the other hand) and a common concern for the subjectivity of our relationship to space enable us to draw them together. The implicit aim of this study is to evidence the specific meanings of "representation" and "landscape" in order to fight the misuse of these words.

\section{INDEX}

Mots-clés : Paysage

\section{AUTEUR}

\section{SOPHIE BONIN}

Maître de conférence, Université Joseph-Fourier, Institut de géographie alpine, Grenoble. Doctorat de géographie, Ladyss, université de Paris 1, sophie.bonin@ujfgrenoble.fr 